\section{Region- and Isoform-Specific Expression of Hydroxysteroid Sulfotransferases in Rat Brain}

\author{
Mikiko Shimizu, ${ }^{a}$ Yukiko Nakano, ${ }^{a}$ \\ Tomoko Yamasaki, ${ }^{a}$ Tetsuya Kimoto, ${ }^{b}$ \\ Suguru Kawato, ${ }^{b}$ and Hiro-omi Tamura*,a \\ ${ }^{a}$ Kyoritsu University of Pharmacy, 1-5-30, Shibakoen, \\ Minatoku, Tokyo 105-8512, Japan and ${ }^{b}$ Department of Biophys- \\ ics and Life Sciences, Graduate School of Arts and Sciences, \\ University of Tokyo at Komaba, 3-8-1 Komaba, Meguro, To- \\ kyo 153-8902, Japan \\ (Received July 29, 2004; Accepted August 19, 2004; \\ Published online August 20, 2004)
}

Hydroxysteroid sulfotransferase (HS-ST) is thought to be a key enzyme in the synthesis of neurosteroid sulfates, which are known to act as potent regulators of neuronal activity within the brain. In rat liver, three isoforms of HS-ST (ST2A1, ST2A2 and ST2A5) have now been identified and to further elucidate the precise contribution of each of these variants in neurosteroid sulfation in rat brain, we analyzed the expression of their transcripts in different brain regions using isoform-specific RT-PCR. The expression of ST2A1 was found exclusively in the olfactory bulb and ST2A2 expression levels were most abundant in the hippocampus, but no ST2A5 expression was detectable in any brain region. We then measured the HS-ST activity in these same regions and detected low but significant activity levels in the olfactory bulb and hippocampus, but less activity in the cortex. These activity levels were found to correlate with the mRNA levels of the HS-ST isoforms. Our findings will further our understanding of the physiological role of HSST enzymes within the brain, particularly how they affect neurosteroid metabolism and modulate neuronal activity.

Key words — brain, neurosteroid, sulfotransferase

\footnotetext{
*To whom all correspondences should be addressed: Kyoritsu University of Pharmacy, 1-5-30, Shibakoen, Minatoku, Tokyo 105-8512, Japan. Tel.: +81-3-5400-2634; Fax: +81-3-54002689; E-mail: tamura-hr@kyoritsu-ph.ac.jp
}

\section{INTRODUCTION}

Neurosteroids, including $3 \beta$-hyroxysteroids such as dehydroepiandrosterone (DHEA) and pregnenolone, and their sulfated esters have been shown to be potent regulators of neuronal activity in the brain $^{1-4)}$ where they modulate the functions of GABA $_{\mathrm{A}}$ receptors $^{3-5)}$ and $N$-methyl- $D$-aspartate (NMDA) receptors. ${ }^{6,7)}$ High levels of DHEA sulfate and pregnenolone sulfate have also been detected in the brain of castrated and adrenalectomized rats, suggesting the presence of sulfation activities in the central nervous system. ${ }^{8)}$

In mammals, the sulfation of $3 \beta$-hydroxysteroids in liver and other tissues is catalyzed by the hydroxysteroid family of sulfotransferases (HS-STs), of which several HS-ST cDNA variants have now been cloned. ${ }^{9,10)}$ In rat liver, 6 isoforms of HS-ST cDNA have been isolated so far; ST2A1 (ST-20), ST2A2 (ST-40), their allelic variants (ST21a, ST21b, and ST41), and ST2A5 (ST-60). Although ST2A1 and ST2A2 share high amino acid homology (> 90\%), ST2A2 displays 20-fold higher DHEA sulfation activity than ST2A1. ${ }^{11)}$ The enzymatic nature of ST2A5 has not yet been characterized. Recently Shimada et al. reported the identification of ST2A1 as a rat brain neurosteroid sulfotransferase, ${ }^{12)}$ although the precise localization of specific HS-ST transcripts in different regions of the brain is still unclear. In this report, we now demonstrate the region specific expression of HS-ST isoforms in rat brain.

\section{MATERIALS AND METHODS}

Brain Tissue — Rat brains were obtained from 5 week old female Sprague-Dawley (SD) rats (100$120 \mathrm{~g}$ ) and dissected into seven predetermined regions. Each sample was immediately frozen at $-80^{\circ} \mathrm{C}$ until use.

RNA Extraction and RT-PCR — Total RNA was isolated from each sample by guanidine thiocyanate phenol-chloroform extraction (Isogen, Nippon Gene Co. Ltd., Tokyo, Japan). The first strand of cDNA was synthesized from $5 \mu \mathrm{g}$ of total RNA using 1 unit M-MLV reverse transcriptase with oligo (dT) primers according to the manufacturer's protocol (Stratagene, Alameda, CA, U.S.A.). Isoform specific PCR primers for HS-STs are as follows ${ }^{13}$ : forward 5'-AGGCCAAGGTGGTCTATCTC-3' and reverse TGATTTTTTCATCAGGAGGC-3' for 
ST2A1, forward 5'-AGGCCAAGGTGGTCTAT CTC-3' and reverse 5'-CAGTTCCTTCTCCATGA GAT- $3^{\prime}$ for ST2A2, and forward $5^{\prime}$-AGGCCAAG GTGGTATATCTC- $3^{\prime}$ and reverse $5^{\prime}$-CAGTTCCT TCTCCATGAGGC- $3^{\prime}$ for ST2A5. The specificity of these primers was determined by direct sequencing of the resulting PCR products. Primers for $\beta$ actin were designed from sequences published in GenBank. PCR amplification of HS-ST mRNAs was performed with AmpliTaq Gold polymerase (Applied Biosystems, Foster City, CA, U.S.A.) under the following conditions: $9 \mathrm{~min}$ at $94^{\circ} \mathrm{C}$, followed by 40 cycles at $94^{\circ} \mathrm{C}$ for $1 \mathrm{~min}, 58^{\circ} \mathrm{C}$ for $1.5 \mathrm{~min}$ and $72^{\circ} \mathrm{C}$ for $2 \mathrm{~min}$.

\section{Preparation of Cytosolic Extracts — Brain} tissues were homogenized in a mixture of $10 \mathrm{mM}$ Tris- $\mathrm{HCl}$ (pH 7.5), $250 \mathrm{mM}$ sucrose, $1 \mathrm{mM}$ dithiothreitol, $5 \mu \mathrm{g} / \mathrm{ml}$ antipine, $5 \mu \mathrm{g} / \mathrm{ml}$ pepstatin and $0.1 \mathrm{mM}$ phenylmethane sulfonyl fluoride (PMSF). The homogenate was then centrifuged at $3000 \mathrm{~g}$ for 10 min and the resulting supernatant was then centrifuged at $105000 \mathrm{~g}$ for $60 \mathrm{~min}$. The final supernatant was used for the assay of HS-ST activity.

Assay of HS-ST Activity - HS-ST activity was determined according to the method of Foldes and Meek with slight modifications. ${ }^{14)}$ Briefly, the reaction mixture contained $20 \mathrm{mM}$ sodium phosphate buffer (pH 6.8), $1 \mu \mathrm{M}\left[{ }^{35} \mathrm{~S}\right] 3^{\prime}$-phosphoadenosine $5^{\prime}$ phosphosulfate (PAPS) $(1 \mu \mathrm{Ci}), 5 \mu \mathrm{M}$ pregnenolone and cytosolic proteins $(0-100 \mu \mathrm{g})$ in a total volume of $250 \mu \mathrm{l}$. The mixtures were incubated at $37^{\circ} \mathrm{C}$ for $30 \mathrm{~min}$ and the reactions were terminated by the addition of $50 \mu \mathrm{l}$ barium acetate. At this stage, unconverted $\left[{ }^{35} \mathrm{~S}\right] \mathrm{PAPS}$ was removed by precipitation with $50 \mu \mathrm{l} 0.1 \mathrm{M} \mathrm{Ba}(\mathrm{OH})_{2}$ and $50 \mu \mathrm{l} 0.1 \mathrm{M} \mathrm{ZnSO}_{4}$. The supernatant $(300 \mu \mathrm{l})$ was then transferred to a $3 \mathrm{ml}$ liquid scintillation vial and the radioactivity levels were counted. Samples without pregnenolone were used as blanks.

\section{RESULTS AND DISCUSSION}

\section{RT-PCR Analysis of HS-ST Expression Levels in Rat Brain}

When RNA was isolated from total brain homogenates of female SD rats and subjected to isoform specific RT-PCR, low but significant levels of ST2A1 expression were detected, consistent with the previous report of Shimada et al. (Fig. 1A). ${ }^{12)}$ To localize the presence of ST2A1 transcripts in the different regions of the rat brain, we subsequently

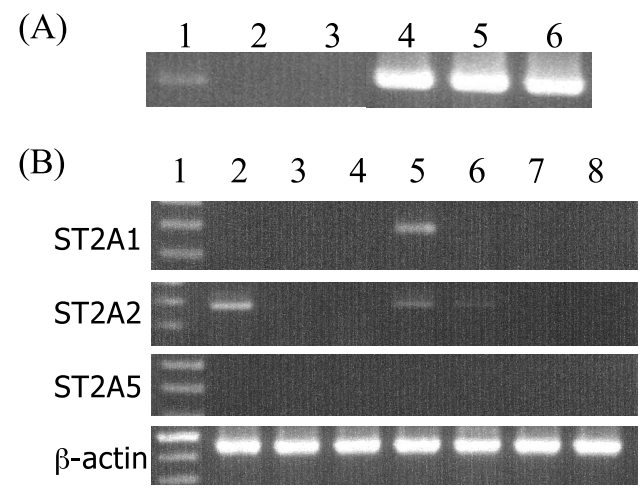

Fig. 1. Isozyme-Specific RT-PCR Analyses of HS-ST Expression Levels in Rat Brain

(A) Total RNA (5 $\mu \mathrm{g})$ isolated from the brain (lane 1-3) and liver (lane 4-6) of female SD rats was subjected to RT-PCR with isozymespecific primers for ST2A1 (lanes 1 and 4), ST2A2 (lanes 2 and 5) and ST2A5 (lanes 3 and 6). The reaction cycles used for brain and liver were 40 and 30, respectively. (B) Region specific expression of HS-ST isozymes in the brain was monitored. Total RNA isolated from each region was subjected to RT-PCR of ST2A1, ST2A2, ST2A5 and $\beta$-actin. Lane 1, size markers; lane 2, hippocampus; lane 3, cortex; lane 4, medulla; lane 5 , olfactory bulb; lane 6 , pons; lane 7 , hypothalamus; lane 8 , cerebellum.

dissected whole brains into 7 distinct regions (cerebellum, cortex, hippocampus, medulla, olfactory bulb, pons and hypothalamus), for individual RTPCR analysis. As shown in Fig. 1B, the expression of ST2A1 was exclusively detected in the olfactory bulb but, interestingly, ST2A2 expression was observed in the hippocampus, olfactory bulb and pons. In contrast, no ST2A5 expression was observed in any of these regions. It is unclear why ST2A2 expression was detectable in our dissected samples and not in the total preparations, but this may be because of a threshold level effect due to specific isolation of a particular region.

\section{HS-ST Activity in the Rat Brain}

HS-ST activity levels are extremely low in rat brain $(0.42 \mathrm{pmol} / \mathrm{min} / \mathrm{mg}$ protein $)$ compared to the levels in rat liver $(0.53 \mathrm{nmol} / \mathrm{min} / \mathrm{mg}$ protein $)$, which are approximately 1000 -fold greater. To evaluate HSST activity in specific regions of the brain, we measured its activity levels in hippocampus, olfactory bulb and cortex. As shown in Fig. 2, higher HS-ST activity was detectable in the olfactory bulb and hippocampus than in the cortex. These results closely correlate with the mRNA expression levels of the HS-ST isozymes in the corresponding regions. Our preliminary immunoblot analysis indicated the presence of $32 \mathrm{kDa}$ proteins cross-reacted with an antiHS-ST antibody in the hippocampus and olfactory 


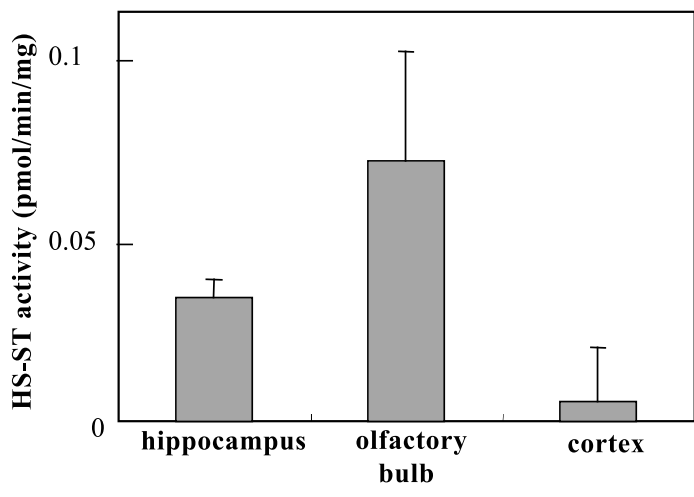

Fig. 2. HS-ST Activity Towards Pregnenolone in Rat Brain HS-ST activities toward $5 \mu \mathrm{M}$ pregnenolone in specific dissected regions of the rat brain (hippocampus, olfactory bulb and cortex) were measured. Cytosolic fractions (50 $\mu \mathrm{g}$ protein) from each dissected area were used in the assay. Bars represent the values of two independent experiments (mean \pm deviation).

bulb cytosols but not in the cortex one (Nakano et al., unpublished data).

In this report we describe the analyses of HSST expression in specific regions of the rat brain and our data demonstrate region specific expression of the HS-ST isozymes ST2A1 and ST2A2 in the olfactory bulb and ST2A2 in the hippocampus and pons. Previously we have demonstrated that an antibody raised against rat liver HS-ST positively immunostained hippocampal neurons. ${ }^{15)}$ The presence of ST2A2 mRNA and pregnenolone sulfating activity in hippocampus strongly suggests that ST2A2 plays a role in neurosteroid sulfoconjugation in the region. Although the role of the extensive expression of HS-ST in the olfactory bulb is unclear, it is tempting to speculate that neurosteroid sulfation regulates the sensitivity of the odor-induced response at this site. Further investigation of the region specific regulation of HS-ST expression in the brain may therefore contribute to our further understanding of the functional role of neurosteroids and their sulfated forms.

Acknowledgements We are grateful to CREST Project on "Endocrine Disruptors in Synthesis and Action of Brain Neurosteroids" of Japan Promotion of Science and Technology.

\section{REFERENCES}

1) Robel, P. and Baulieu, E. E. (1995) Dehydroepiandrosterone (DHEA) is a neuroactive neurosteroid.
Ann. N.Y. Acad. Sci., 774, 82-110.

2) Baulieu, E. E. (1998) Neurosteroids: a novel function of the brain. Psychoneuroendocrinology, 23, 963-987.

3) Majewska, M. D., Harrison, N. L., Schwartz, R. D., Barker, J. L. and Paul, S. M. (1998) Steroid hormone metabolites are barbiturate-like modulators of the GABA receptor. Science, 232, 1004-1007.

4) Compagnone, N. A. and Mellon, S. H. (2000) Neurosteroids: biosynthesis and function of these novel neuromodulators. Front. Neuroendocrinol., 21, 1-56.

5) Wu, F. S., Gibbs, T. T. and Farb, D. H. (1990) Inverse modulation of gamma-aminobutyric acid- and glycine-induced currents by progesterone. $\mathrm{Mol}$. Pharmacol., 37, 597-602.

6) Partridge, L. D. and Valenzuela, C. F. (2001) Neurosteroid-induced enhancement of glutamate transmission in rat hippocampal slices. Neurosci. Lett., 301, 103-106.

7) Wu, F. S., Gibbs, T. T. and Farb, D. H. (1991) Pregnenolone sulfate: a positive allosteric modulator at the $N$-methyl- $D$-aspartate receptor. $M o l$. Pharmacol., 40, 333-336.

8) Corpechot, C., Robel, P., Axelson, M., Sjovall, J. and Baulieu, E. E. (1981) Characterization and measurement of dehydroepiandrosterone sulfate in rat brain. Proc. Natl. Acad. Sci. U.S.A., 78, 4704-4707.

9) Weinshilboum, R. and Otterness, D. (1994) Sulfotransferase enzymes. In Conjugation-deconjugation Reactions in Drug Metabolism and Toxicity (Kauffman, F. C., Ed.), Springer-Verlag, Berlin, pp. 45-78.

10) Nagata, K. and Yamazoe, Y. (2000) Pharmacogenetics of sulfotransferase. Ann. Rev. Pharmacol. Toxicol., 40, 159-176.

11) Tamura, H., Morioka, Y., Homma, H. and Matsui, M. (1997) Construction and expression of chimeric rat liver hydroxysteroid sulfotransferase isozymes. Arch. Biochem. Biophys., 341, 309-314.

12) Shimada, M., Yoshinari, K., Tanabe, E., Shimakawa, E., Kobashi, M., Nagata, K. and Yamazoe, Y. (2001) Identification of ST2A1 as a rat brain neurosteroid sulfotransferase mRNA. Brain Res., 920, 222-225.

13) Takahashi, M., Tamura, H., Kondo, S., Kobayashi, K. and Matsui, M. (1998) Identification of three hydroxysteroid sulfotransferase isoenzymes in the rat liver. Biol. Pharm. Bull., 21, 10-15.

14) Foldes, A. and Meek, J. L. (1973) Rat brain phenolsulfotransferase; Partial purification and some properties. Biochim. Biophys. Acta, 327, 365-374.

15) Kimoto, T., Tsurugizawa, T., Ohta, Y., Makino, J., Tamura, H., Hojo, Y., Takata, N. and Kawato, S. (2001) Neuristeroid synthesis by cytochrome P450- 
containing systems localized in the rat brain hippocampal neurons: $N$-methyl- $D$-aspartate and cal- cium-dependent synthesis. Endocrinology, 142, 3578-3589. 